http://dx.doi.org/10.32911/as.2018.v11.n2.578

Aporte Santiaguino. 11(2), julio-diciembre 2018: 237-250

ISSN-L 2616-9541

\title{
Eficiencia de la Unidad Básica de Saneamiento empleando humedales artificiales con especies nativas para la depuración de aguas residuales en el centro experimental Tuyu Ruri - Marcara, para su reúso como agua de riego
}

Efficiency of the Basic Sanitation Unit using artificial wetlands with native species for the purification of wastewater in the Tuyu Ruri - Marcara experimental center, for irrigation water reuse

\author{
Rosario Adriana Polo Salazar ${ }^{1}$, Martín Miguel Huamán Carranza ${ }^{1}$, Judith Isabel \\ Flores Albornoz ${ }^{1}$, Carlos Borromeo Poma Villafuerte ${ }^{1} \mathrm{Y}$ \\ César Manuel Dávila Paredes ${ }^{1}$
}

\section{RESUMEN}

El trabajo de investigación tuvo por objetivo evaluar la eficiencia de la Unidad Básica de Saneamiento (UBS) empleando humedales artificiales con especies nativas de la zona, se implementó un Biodigestor y se construyó dos humedales: uno con Cola de Caballo y otro con Cartucho, así mismo evaluarlas para su reúso como agua de riego. Las muestras se obtuvieron de la caja de recepción, de las salidas del: Biodigestor, humedal con Cartucho y humedal con Cola de caballo. La eficiencia de remoción de la Demanda Bioquímica de Oxígeno (DBO) fue 82\% y la Demanda Química de Oxígeno (DQO) fue 81\%, ambos en la salida del Biodigestor. La remoción en la Demanda Bioquímica de Oxígeno (DBO) en la salida del humedal con Cartucho fue 68\% y del humedal con Cola de caballo fue 58\%; de la Demanda Química de Oxígeno (DQO) en el humedal con Cartucho fue 68\% y del humedal con Cola de caballo fue 58\%. Los resultados obtenidos de las diversas salidas evaluadas se compararon con los Estándares de Calidad Ambiental (ECA), como: la Demanda Bioquímica de Oxígeno (DBO), De-

\footnotetext{
1 Universidad Nacional Santiago Antúnez de Mayolo. Huaraz, Perú.

(C) Los autores. Este artículo es publicado por la Revista Aporte Santiaguino de la Universidad Nacional Santiago Antúnez de Mayolo. Este es un artículo de acceso abierto, distribuido bajo los términos de la Licencia Creative Commons Atribución-NoComercial-CompartirIgual 4.0 Internacional. (http://creativecommons.org/licenses/ by-nc-sa/4.0/), que permite el uso no comercial, distribución y reproducción en cualquier medio, siempre que la obra original sea debidamente citada.
} 
manda Química de Oxígeno (DQO), los coliformes totales y fecales, el Fosfato $\left(\mathrm{PO}_{4}\right.$ ) y Nitrato $\left(\mathrm{NO}_{3}\right)$ mientras que los Sólidos Suspendidos Totales (SST) fueron comparados con la Norma Chilena de DIGESA.

Palabras clave: contaminación; aguas residuales; humedales artificiales; calidad del agua.

\section{ABSTRACT}

The objective of the research work was to evaluate the efficiency of the Basic Sanitation Unit (UBS) using artificial wetlands with native species from the area, a Biodigestor was implemented and two wetlands were built: one with Horsetail and another with Cartridge, as well as same evaluate them for their reuse as irrigation water. The samples were obtained from the reception box, from the outputs of: Biodigestor, wetland with Cartridge and wetland with Horsetail. The removal efficiency of the Biochemical Oxygen Demand (BOD) was $82 \%$ and the Chemical Oxygen Demand (COD) was $81 \%$, both at the output of the Biodigestor. The removal in the Biochemical Oxygen Demand (BOD) at the exit of the wetland with Cartridge was 68\% and the wetland with Horsetail was $58 \%$; of the Chemical Oxygen Demand (COD) in the wetland with Cartridge was $68 \%$ and of the wetland with Horsetail was $58 \%$. The results obtained from the various outputs evaluated were compared with the Environmental Quality Standards (ECA), such as: Biochemical Oxygen Demand (BOD), Chemical Oxygen Demand (COD), total and fecal coliforms, Phosphate $\left(\mathrm{PO}_{4}\right)$ and Nitrate $\left(\mathrm{NO}_{3}\right)$ while the Total Suspended Solids (SST) were compared with the Chilean Standard of DIGESA.

Keywords: pollution; wastewater; artificial wetlands; water quality.

\section{INTRODUCCIÓN}

El trabajo de investigación evalúa la eficiencia de la unidad básica de saneamiento empleando humedales artificiales para la depuración de aguas residuales en el Centro Experimental de Tuyu Ruri; aportando en la conservación del agua y reusarla en riego sin riesgo alguno, sobre todo en zonas rurales y comunidades pequeñas.

Delgadillo et al (2010) manifiesta "En la actualidad existe una gran presión sobre los recursos hídricos a nivel mundial; según la UNESCO (2003) el 69\% del agua dulce disponible en el planeta se destina a la agricultura, el 23\% a la industria y el $8 \%$ al consumo doméstico".

Perdana (2018) manifesto "Este estudio experimental aplicó cuatro tratamientos: 1) 
control (sin plantar), 2)especies individuales Iris pseudacorus, 3)especies únicas Echinodorus palaefolius, y 4)Combinación (Iris pseudacorus y Echinodorus palaefolius) con tres días de tiempo de retención. El resultado mostró que el valor promedio de efectividad para los parámetros fueron: DQO por tratamiento combinado $(50,76 \%)$, $\mathrm{DBO}_{5}$ solo por I. pseudacorus $(30.15 \%)$, nitrato por E. palaefolius $(58,06 \%)$, fosfato por E. palaefolius (99,5\%), y TDS por E. palaefolius (3,25\%). El resultado mostró que había una diferencia significativa de la reducción de nitrato y fosfato entre el control y los otros tres tratamientos".

Vymazal (2015) en su trabajo de investigación titulado "Accumulation of heavy metals in aboveground biomass of Phragmites australis in horizontal flow constructed wetlands for wastewater treatment: A review" manifiesta "El tratamiento de aguas residuales en humedales construidos es un proceso biotecnológico que se ha utilizado durante más de cinco décadas para el tratamiento de aguas residuales. En general, manifiesta que las plantas son una parte importante del sistema de tratamiento; sin embargo, el papel directo de las plantas se restringe a la absorción de nutrientes y metales pesados de las plantas. El propósito de este estudio fue evaluar la cantidad de metales pesados secuestrados en la biomasa aérea de Phragmites australis, por lo tanto, disponible para la recolección y remoción. La cantidad de metales pesados secuestrados en los brotes de la planta como una fracción del metal pesado total eliminado en el humedal construido es variable con valores tan altos como $71 \%$ para cadmio, 55\% para cromo o $49 \%$ para zinc en algunos estudios".

En el ámbito profesional y académico se desea proponer y evaluar el funcionamiento de las Unidades Básicas de Saneamiento (UBS) con los humedales artificiales empleando plantas de la zona como la Cola de caballo y el Cartucho, con la finalidad de aportar y profundizar los conocimientos en la aplicación de Biotecnologías sencillas, disminuyendo los efectos negativos de las aguas residuales al ambiente y obtener aguas tratadas para destinarlas a las áreas de cultivo sin riesgo alguno. Esto ayudará a generar nuevas tecnologías en expedientes técnicos de agua y saneamiento especialmente en zonas rurales de Ancash y puedan ser implementados por las municipalidades sobre todo en poblados rurales con un déficit grande de cobertura de servicios de saneamiento.

\section{MATERIALES Y MÉTODOS}

En el trabajo se evaluó la eficiencia en el uso de humedales con plantas de la zona junto a Unidades Básicas de Saneamiento (UBS) para el reúso del agua en riego, empleando tecnologías con fines de recuperar el agua residual y remover la materia orgánica de las aguas residuales mediante procesos químicos, físicos y microbiológicos; para un mejor funcionamiento en los humedales se utilizaron plantas de la zona: el Cartucho y la Cola de Caballo. 
La investigación es Aplicada y Experimental y el diseño es Cuasi experimental. La Población es el centro experimental Tuyu Ruri, y la muestra es el agua residual. Para el desarrollo de la investigación se utilizó un biodigestor como tratamiento primario y humedales, uno con Cartucho y otro con Cola de Caballo, ambos como tratamiento secundario. La metodología a seguir fue la instalación y funcionamiento del sistema. Se determinó 04 puntos de muestreo: el ingreso del agua residual en la caja de recepción (punto 1), la salida del Biodigestor (punto 2), la salida del humedal con Cartucho (punto 3) y la salida del humedal con Cola de caballo (punto 4), para analizar: Demanda Bioquímica de Oxígeno (DBO), Demanda Química de Oxígeno (DQO), Sólidos Suspendidos Totales (SST), Coliformes Totales, Coliformes Fecales, Fosfato $\left(\mathrm{PO}_{4}\right)$, Sulfato $\left(\mathrm{SO}_{4}\right)$, Amonio $\left(\mathrm{NH}_{4}\right)$, Nitrito $\left(\mathrm{NO}_{2}\right)$ y Nitrato $\left(\mathrm{NO}_{3}\right)$, con los cuales se determinó la eficiencia del tratamiento.

\section{RESULTADOS}

Para evaluar el porcentaje de remoción del Biodigestor, Humedal con Cola de Caballo y Humedal con Cartucho, se evaluó los parámetros en la entrada y en la salida de cada unidad mencionada cuyos resultados se muestran en las tablas 1, 2 y 3 :

Tabla 1. Resultados del agua residual que ingresa y la sale del biodigestor

\begin{tabular}{|c|c|c|c|c|c|c|}
\hline Muestreo & $\begin{array}{l}\text { Punto de } \\
\text { muestreo }\end{array}$ & $\begin{array}{l}\mathrm{DBO} \\
(\mathrm{mg} / \mathrm{l})\end{array}$ & $\begin{array}{l}\text { DQO } \\
(\mathrm{mg} / \mathrm{l})\end{array}$ & $\begin{array}{c}\mathrm{SST} \\
(\mathrm{mg} / \mathrm{l})\end{array}$ & $\begin{array}{c}\text { Coliformes } \\
\text { Totales } \\
\text { (NMP/100ml) }\end{array}$ & $\begin{array}{c}\text { Coliformes } \\
\text { Fecales } \\
(\mathrm{NMP} / 100 \mathrm{ml})\end{array}$ \\
\hline \multirow{2}{*}{ M1 } & E-1 & 132 & 263 & 67 & 24000000 & 11000000 \\
\hline & S-1 & 26 & 52 & 4 & 24000000 & 11000000 \\
\hline \multirow{2}{*}{ M2 } & E-2 & 19 & 114 & 51 & 24000000 & 11000000 \\
\hline & S-2 & 28 & 127 & 3 & 24000000 & 11000000 \\
\hline \multirow{2}{*}{ M3 } & E-3 & 13 & 26 & 15 & 11000000 & 4600000 \\
\hline & S-3 & 50 & 100 & 5 & 2400000 & 1100000 \\
\hline \multirow{2}{*}{ M4 } & E-4 & 94 & 102 & 132 & 24000000 & 11000000 \\
\hline & S-4 & 46 & 92 & 7 & 24000000 & 11000000 \\
\hline \multirow{2}{*}{ M5 } & E-5 & 33 & 185 & 216 & 24000000 & 11000000 \\
\hline & S-5 & 55 & 100 & 11 & 24000000 & 11000000 \\
\hline \multirow{2}{*}{ M6 } & E-6 & 66 & 179 & 247 & 24000000 & 11000000 \\
\hline & S-6 & 22 & 117 & 10 & 24000000 & 11000000 \\
\hline \multirow{2}{*}{ M7 } & E-7 & 293 & 318 & 117 & 24000000 & 11000000 \\
\hline & S-7 & 148 & 161 & 9 & 24000000 & 11000000 \\
\hline \multirow{2}{*}{ M8 } & E-8 & 142 & 355 & 171 & 24000000 & 11000000 \\
\hline & S-8 & 45 & 103 & 7 & 24000000 & 11000000 \\
\hline \multirow{2}{*}{ M9 } & E-9 & 137 & 396 & 245 & 24000000 & 11000000 \\
\hline & S-9 & 158 & 454 & 46 & 24000000 & 11000000 \\
\hline \multirow{2}{*}{ M10 } & E-10 & 180 & 342 & 161 & 24000000 & 11000000 \\
\hline & S-10 & 33 & 66 & 47 & 24000000 & 11000000 \\
\hline
\end{tabular}


Tabla 2. Resultados del agua tratada que entra y sale del humedal con cola de caballo

\begin{tabular}{ccccc}
\hline \multirow{2}{*}{ Muestreo } & $\begin{array}{c}\text { Punto de } \\
\text { muestreo }\end{array}$ & $\begin{array}{c}\text { DBO } \\
(\mathrm{mg} / \mathrm{l})\end{array}$ & $\begin{array}{c}\text { Coliformes Totales } \\
(\mathrm{NMP} / 100 \mathrm{ml})\end{array}$ & $\begin{array}{c}\text { Coliformes Fecales } \\
\text { (NMP/100ml })\end{array}$ \\
\hline \multirow{2}{*}{ M1 } & E-1 & 33 & 24000000 & 11000000 \\
& S-1 & 10 & 2400000 & 1100000 \\
M2 & E-2 & 37 & 24000000 & 11000000 \\
& S-2 & 15 & 24000000 & 11000000 \\
M3 & E-3 & 50 & 2400000 & 1100000 \\
& S-3 & 21 & 2400000 & 1100000 \\
M4 & E-4 & 29 & 24000000 & 11000000 \\
& S-4 & 6 & 24000000 & 11000000 \\
M5 & E-5 & 46 & 24000000 & 11000000 \\
& S-5 & 23 & 24000000 & 41000000 \\
M6 & E-6 & 91 & 24000000 & 2400000 \\
& S-6 & 20 & 24000000 & 11000000 \\
M7 & E-7 & 120 & 24000000 & 11000000 \\
& S-7 & 11 & 24000000 & 11000000 \\
& E-8 & 48 & 24000000 & 11000000 \\
\hline
\end{tabular}

Tabla 3. Resultados del agua tratada que entra y sale del humedal con cartucho

\begin{tabular}{ccccccc}
\hline \multirow{2}{*}{ Muestreo } & $\begin{array}{c}\text { Punto de } \\
\text { muestreo }\end{array}$ & $\begin{array}{c}\mathrm{DBO} \\
(\mathrm{mg} / \mathrm{l})\end{array}$ & $\begin{array}{c}\mathrm{SST} \\
(\mathrm{mg} / \mathrm{l})\end{array}$ & $\begin{array}{c}\mathrm{PO}_{4} \\
(\mathrm{mg} / \mathrm{l})\end{array}$ & $\begin{array}{c}\mathrm{NH}_{4} \\
(\mathrm{mg} / \mathrm{l})\end{array}$ & $\begin{array}{c}\mathrm{NO}_{3} \\
(\mathrm{mg} / \mathrm{l})\end{array}$ \\
\hline \multirow{2}{*}{ M1 } & E-1 & 40 & 1 & 2,29 & 14,00 & 1,0 \\
& S-1 & 38 & 6 & 1,00 & 6,90 & 1,0 \\
\multirow{2}{*}{ M2 } & E-2 & 33 & 1 & 3,05 & 19,60 & 1,0 \\
& S-2 & 17 & 1 & 0,69 & 4,40 & 1,0 \\
M3 & E-3 & 50 & 5 & 0,79 & 15,60 & 1,2 \\
& S-3 & 16 & 2 & 0,74 & 5,70 & 1,0 \\
M4 & E-4 & -------- & 11 & 0,51 & 0,010 & 6,1 \\
& S-4 & -------- & 5 & 0,49 & 0,010 & 3,8 \\
M5 & E-5 & -------- & 6 & 0,20 & 0,119 & 7,3 \\
& S-5 & -------- & 3 & 0,05 & 0,083 & 5,1 \\
M6 & E-6 & -------- & 3 & 7,80 & 0,447 & 7,1 \\
& S-6 & -------- & 3 & 7,90 & 0,514 & 3,8 \\
\multirow{2}{*}{ M7 } & E-7 & 40 & 9 & 0,17 & 1,880 & 1,9 \\
& S-7 & 32 & 11 & 0,17 & 1,110 & 1,8 \\
\hline
\end{tabular}




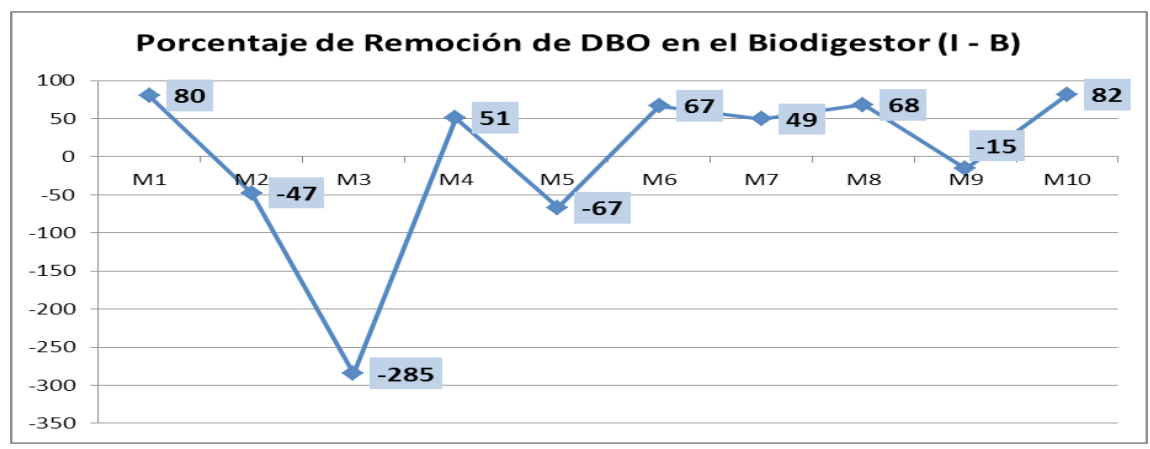

Figura 1. Porcentaje de remoción de la $\mathrm{DBO}_{5}$ en la salida del biodigestor

La figura 1 muestra la remoción de la $\mathrm{DBO}_{5}$ en la salida del Biodigestor, siendo fluctuantes; teniendo porcentajes altos y negativos; por la lenta adaptación de los microorganismos.

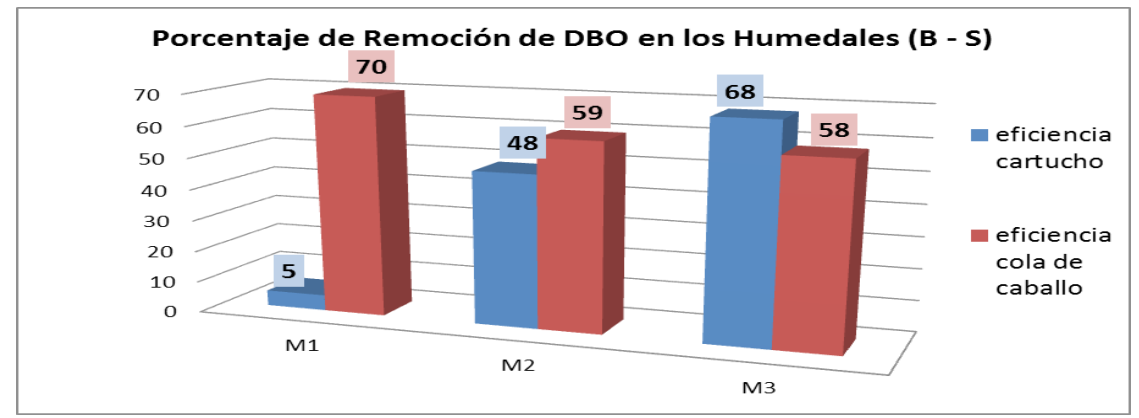

Figura 2. Porcentaje de remoción de $\mathrm{DBO}_{5}$ en la salida de los humedales

La figura 2 muestra los porcentajes de remoción de la $\mathrm{DBO}_{5}$, donde el humedal con Cartucho presenta mayor remoción que el humedal con Cola de caballo mostrando una remoción baja al final del trabajo.

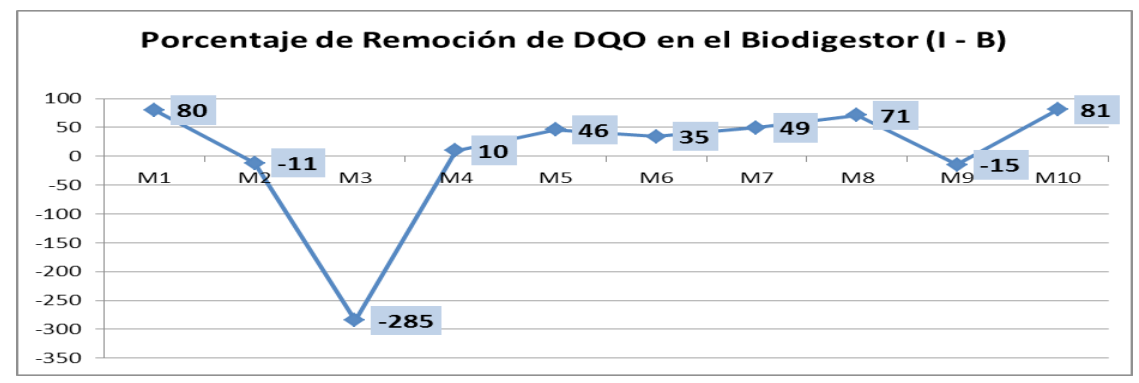

Figura 3. Porcentaje de remoción de DQO en la salida del biodigestor

La figura 3 muestra la remoción de la DQO en la salida del Biodigestor, siendo fluctuante; primero bajos por el lento proceso químico y posteriormente se recupera. 


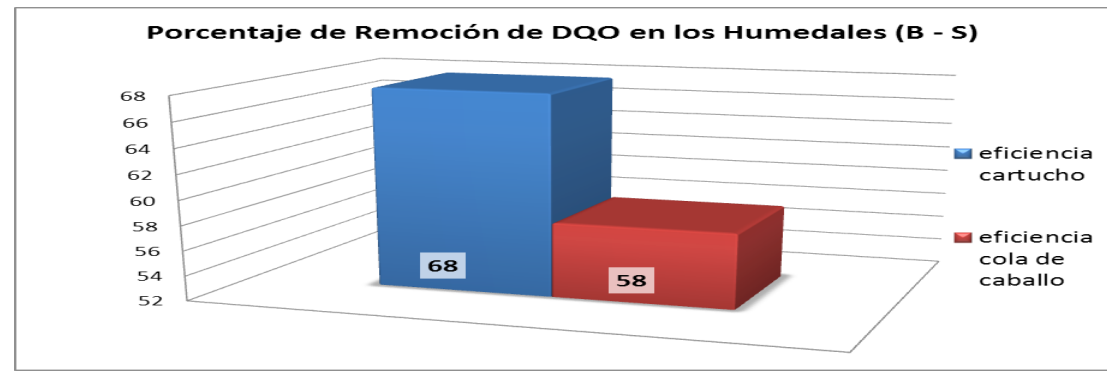

Figura 4. Porcentaje de remoción de DQO en la salida de los humedales

La figura 4 muestra porcentajes de remoción de la DQO, donde el humedal con Cartucho tiene mayor remoción que el humedal con Cola de caballo cuya remoción es buena, pero lenta.

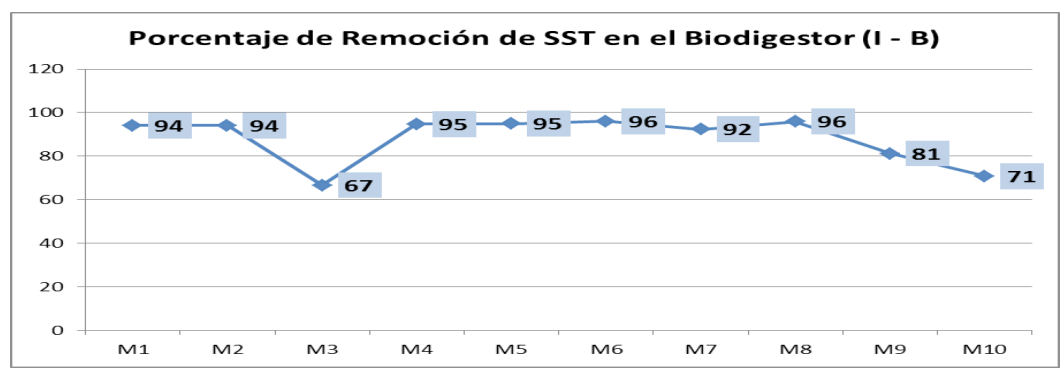

Figura 5. Porcentaje de remoción de SST en la salida del biodigestor

La figura 5 muestra la remoción de SST siendo eficiente y fluctuante por los procesos fisicoquímicos lentos del biodigestor.

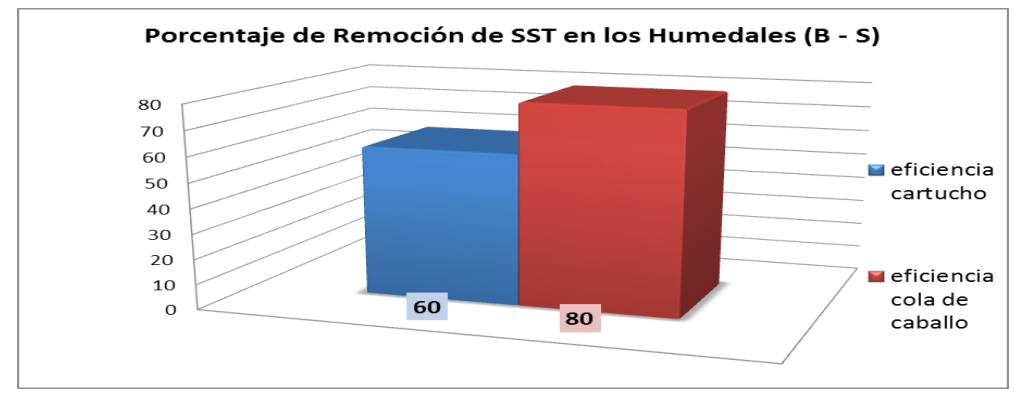

Figura 6. Porcentaje de remoción de SST en la salida de los humedales

La figura 6 muestra porcentajes de remoción de SST, donde el humedal con Cola de caballo presenta una mayor remoción de SST que el humedal con Cartucho cuya remoción es menor. 


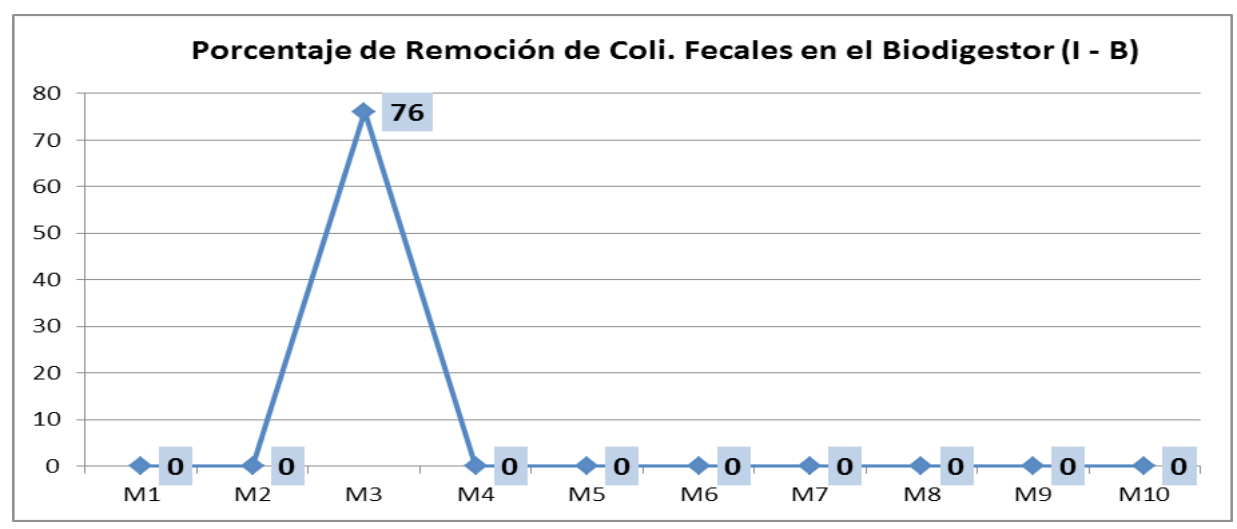

Figura 7. Porcentaje de remoción de coliformes fecales en la salida del biodigestor

La figura 7 muestra que no se remueve coliformes fecales en la mayoría de los muestreos; ya que ellos son los responsables de la degradación de la materia orgánica.

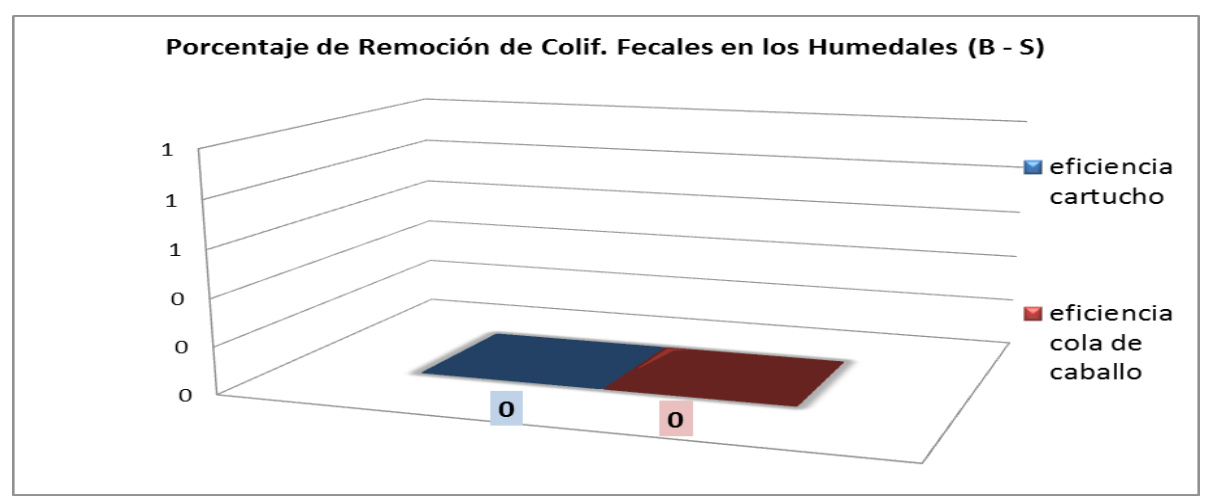

Figura 8. Porcentaje de remoción de coliformes fecales en la salida de los humedales

La figura 8 muestra que no se remueve coliformes fecales en la salida de los humedales, ya que ellos realizan la degradación de la materia orgánica.

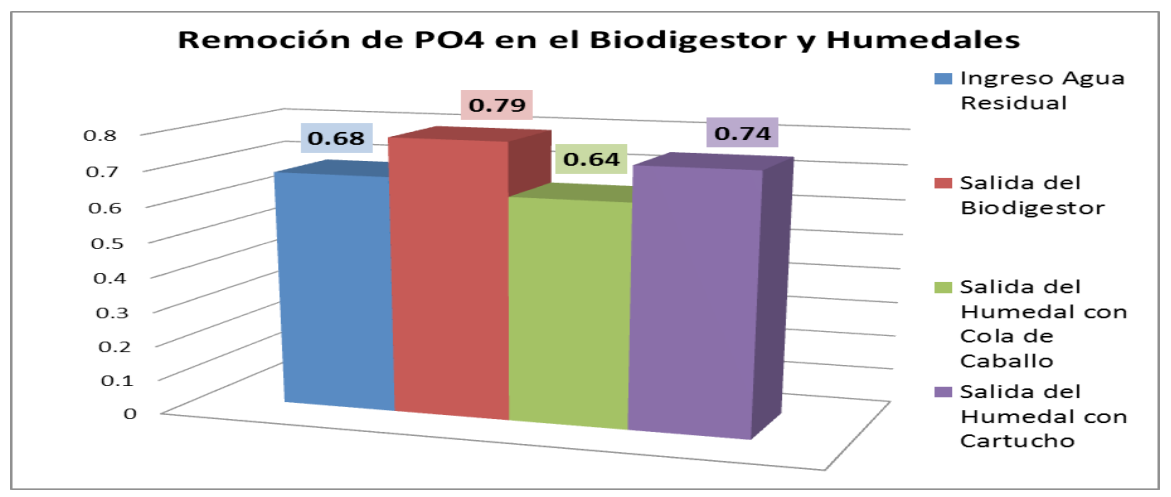

Figura 9. Remoción de $\mathrm{PO}_{4}(\mathrm{mg} / \mathrm{l})$ en la salida del biodigestor y humedales 
La figura 9 muestra la remoción de $\mathrm{PO}_{4}$, bajo en la entrada, se incrementa en la salida del biodigestor, en la salida del humedal con Cola de caballo disminuye por la asimilación en las plantas y en la salida del humedal con Cartucho aumenta por la asimilación es lenta.

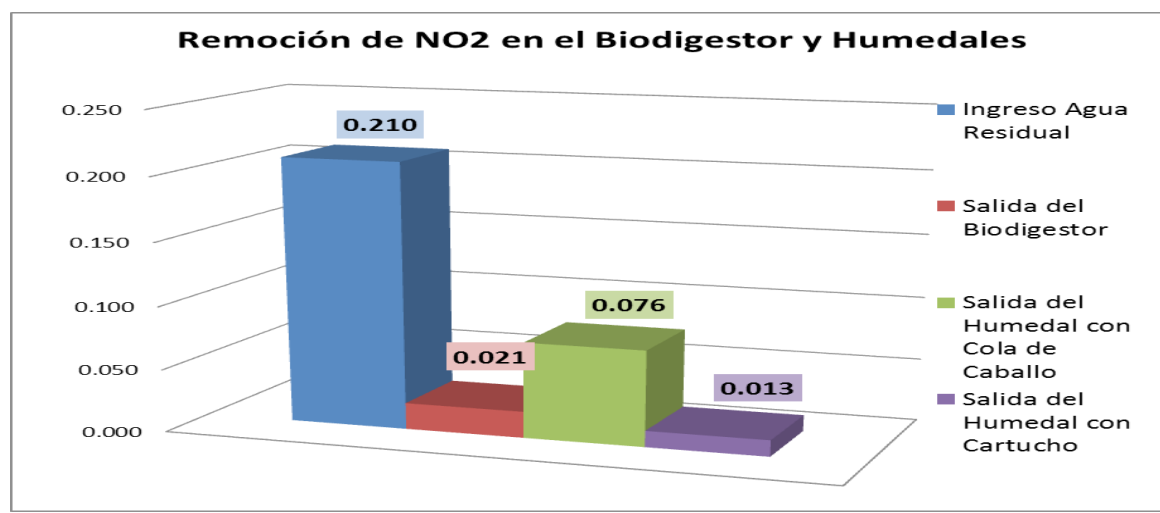

Figura 10. Remoción de $\mathrm{NO}_{2}(\mathrm{mg} / \mathrm{l})$ en la salida del biodigestor y humedales

La figura 10 muestra la remoción de $\mathrm{NO}_{2}$, alto en la entrada, en la salida del biodigestor disminuye por el proceso de desnitrificación, en el humedal con Cola de caballo aumenta por el proceso de oxidación y en el humedal con Cartucho baja por el proceso de oxidación lento.

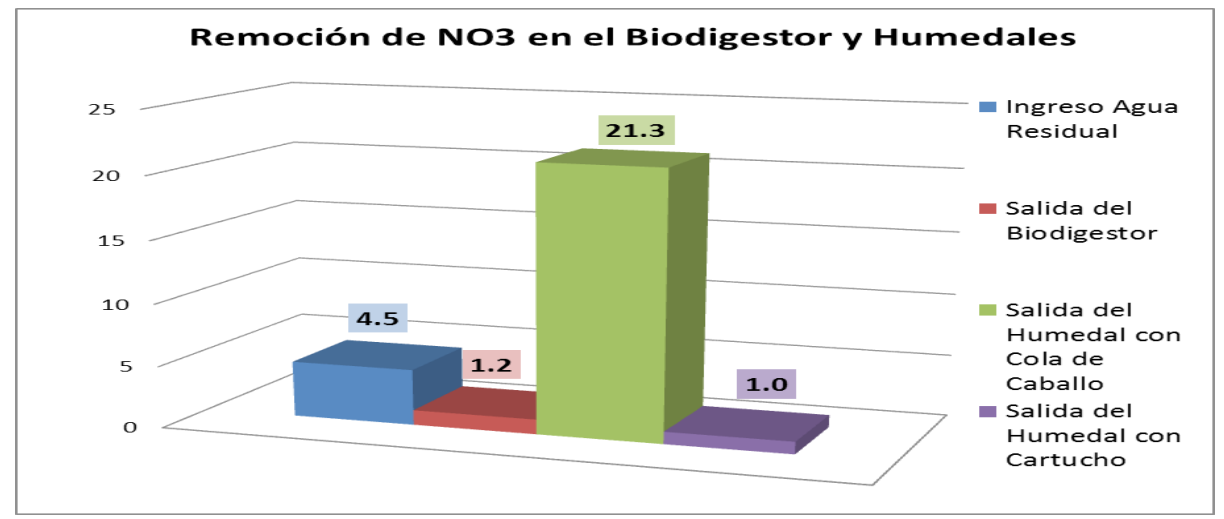

Figura 11. Remoción de $\mathrm{NO}_{3}(\mathrm{mg} / \mathrm{l})$ en la salida del biodigestor y humedales

La figura 11 muestra la remoción de $\mathrm{NO}_{3}$, en la entrada presenta valores bajos, en la salida del biodigestor disminuye por la desnitrificación; en el humedal con Cola de caballo aumenta por el proceso de oxidación y en el humedal con Cartucho baja por el proceso de oxidación lento. 


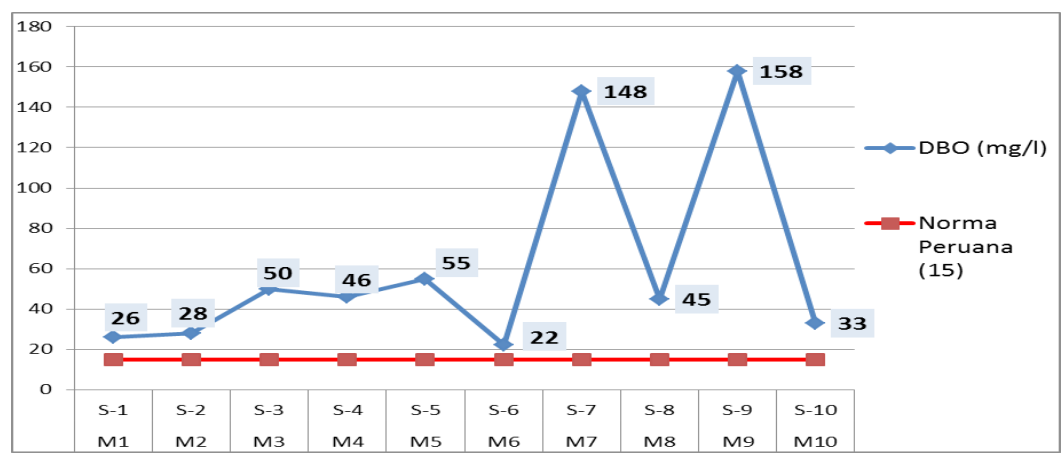

Figura 12. Comparación de la $\mathrm{DBO}_{5}$ en la salida del biodigestor con las ECAs

La figura 12 muestra los resultados de $\mathrm{DBO}_{5}$ de la salida del biodigestor que están por encima de la norma cuyo valor aceptable es $15 \mathrm{mg} / \mathrm{l}$, e indica que el agua que sale del Biodigestor contiene materia orgánica.

Los resultados se compararon con el Decreto Supremo (2017) "Estándares de calidad ambiental para Agua y establecen disposiciones complementarias" y los "Estándares de calidad ambiental de grupo $\mathrm{N}^{\circ}$ 3: Riego de Vegetales y bebida de animales" de DIGESA (2008).

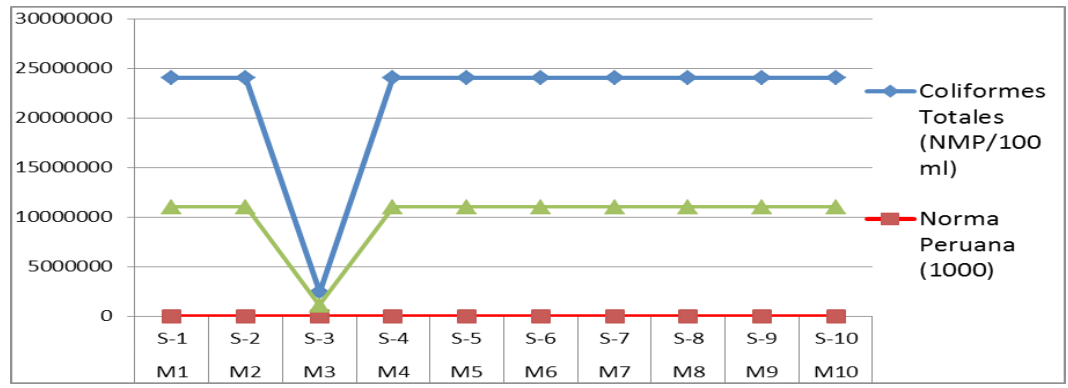

Figura 13. Comparación de los colif. totales en la salida del biodigestor con las ECAs

La figura 13 muestra los resultados de coliformes totales en la salida del Biodigestor, que están por encima de la norma, cuyo valor aceptable es $1000 \mathrm{NMP} / 100 \mathrm{ml}$; ya que el Biodigestor no es una unidad de tratamiento que elimine microorganismos.

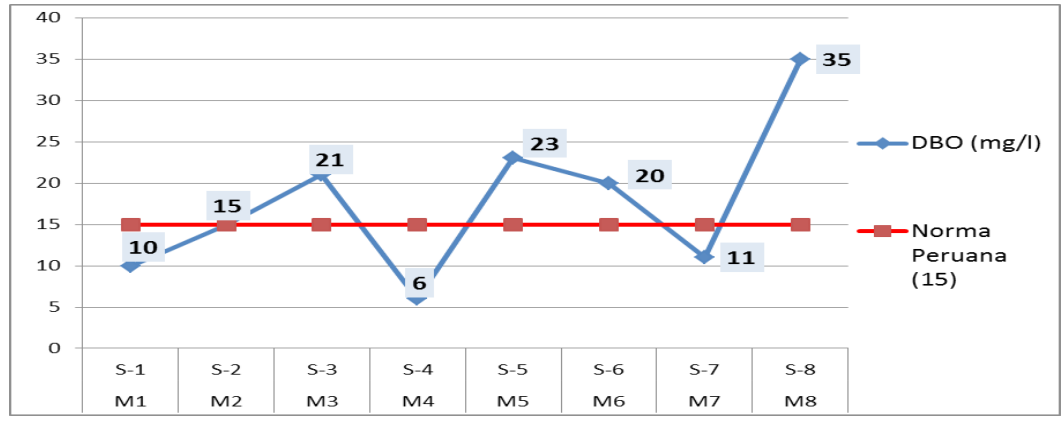

Figura 14. Comparación de $\mathrm{DBO}_{5}$ del humedal con Cola de caballo con las ECAs 
La figura 14 muestra la remoción de la $\mathrm{DBO}_{5}$ en la salida del humedal con Cola de caballo, los resultados están por debajo y encima de la norma, cuyo valor aceptable es $15 \mathrm{mg} / 1$; por la materia orgánica no puede ser retenida ni degradada.

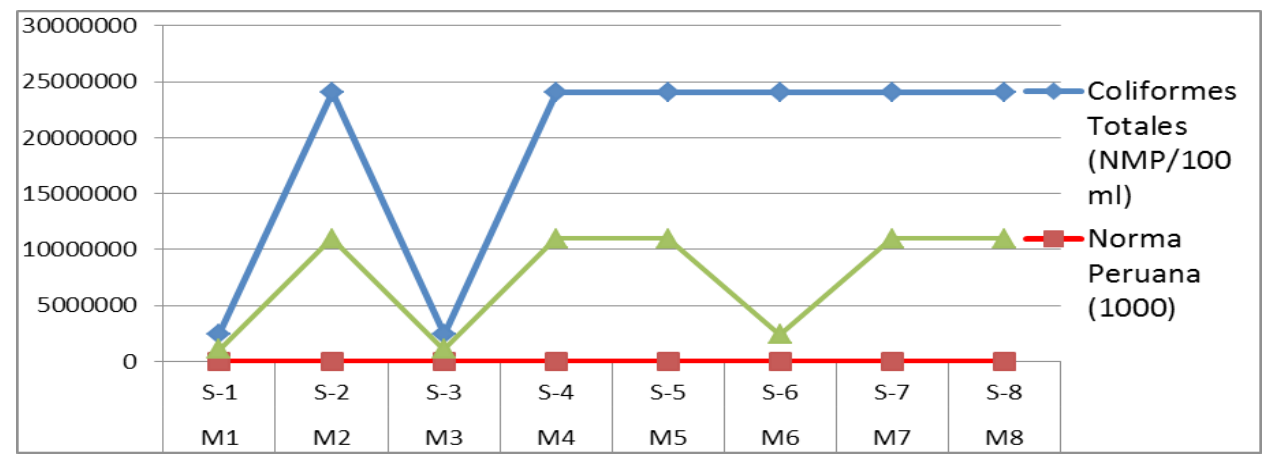

Figura 15. Comparación de colif. totales del humedal con Cola de caballo con la ECAs

La figura 15 muestra la remoción de Coliformes Totales en la salida del humedal con Cola de caballo cuyos resultados están por encima de la norma, siendo el valor aceptable 1000NMP/100 ml; ya que el humedal no remueve ni elimina microorganismos.

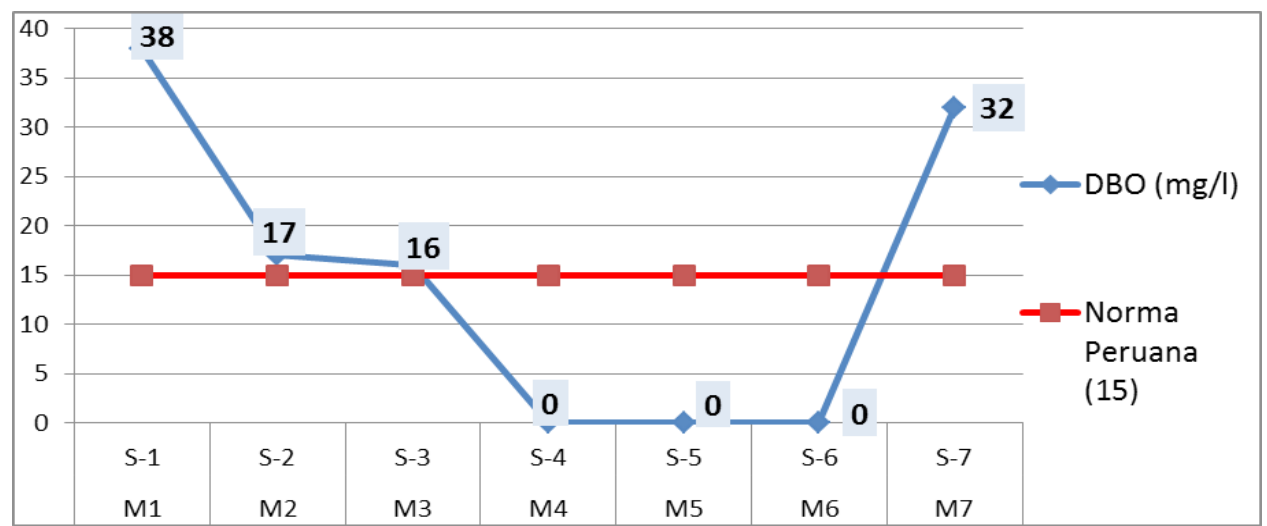

Figura 16. Comparación de la $\mathrm{DBO}_{5}$ del humedal con Cartucho con las ECAs

La figura 16 muestra la remoción de la $\mathrm{DBO}_{5}$ en la salida del humedal con Cartucho, cuyos resultados están por debajo y otros por encima de la norma, cuyo valor aceptable es $15 \mathrm{mg} / \mathrm{l}$; debido a la presencia de materia orgánica que el humedal no puede retener ni degradar. 


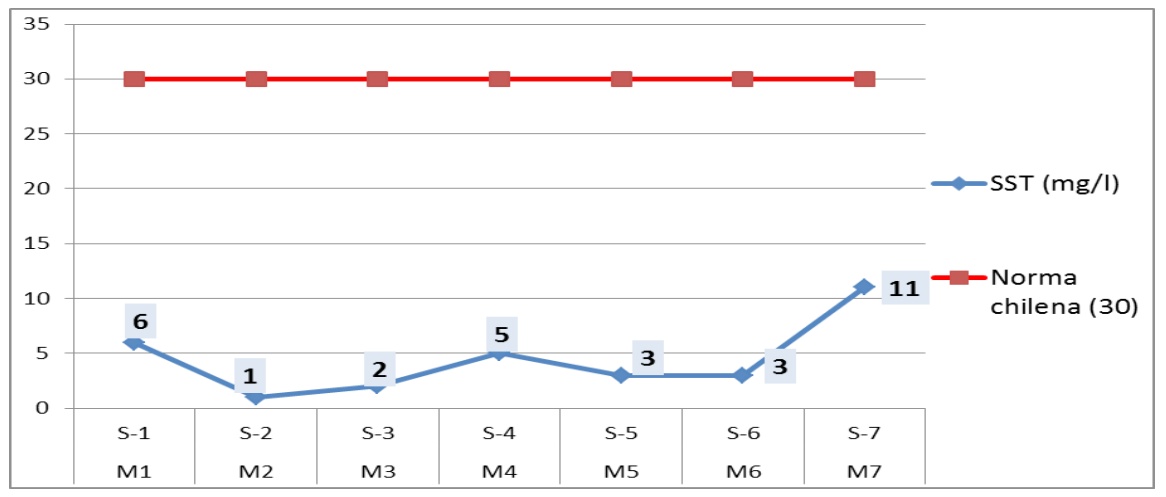

Figura 17. Comparación de SST en la salida del humedal con Cartucho con las ECAs

La figura 17 muestra la remoción de SST en la salida del humedal con Cartucho cuyos resultados están por debajo de la norma, cuyo valor aceptable es $30 \mathrm{mg} / \mathrm{l}$; lo que indica que las plantas del humedal retienen los sólidos presentes en el agua.

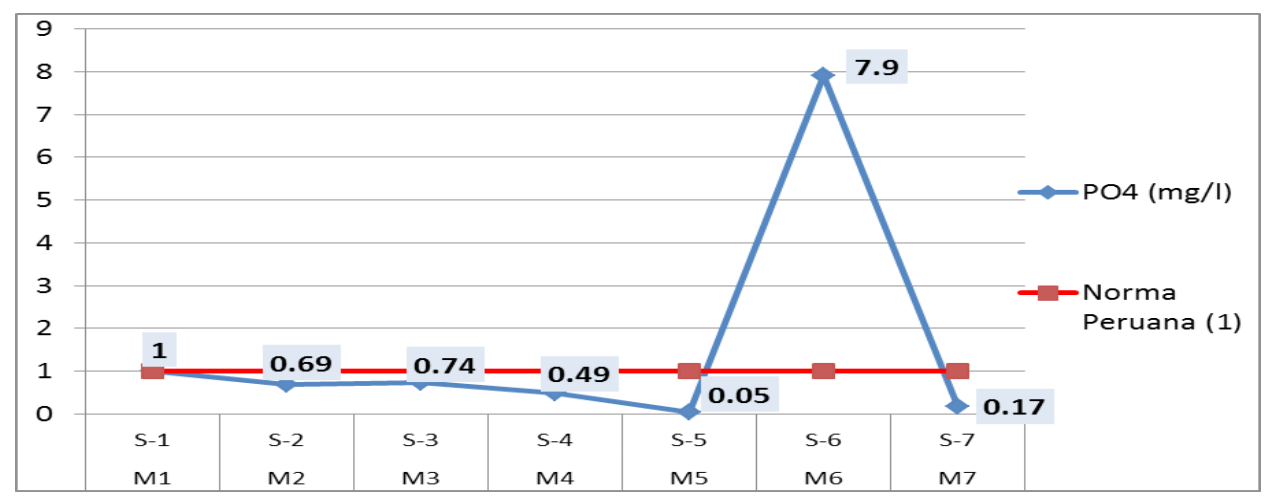

Figura 18. Comparación de $\mathrm{PO}_{4}$ en la salida del humedal con Cartucho con las ECAs

La figura 18 muestra la remoción de $\mathrm{PO}_{4}$ en la salida del humedal con Cartucho, donde los resultados están por debajo de la norma, cuyo valor aceptable es $1 \mathrm{mg} / \mathrm{l}$; lo que indica que las plantas a nivel de la raíz están asimilando el $\mathrm{PO}_{4}$.

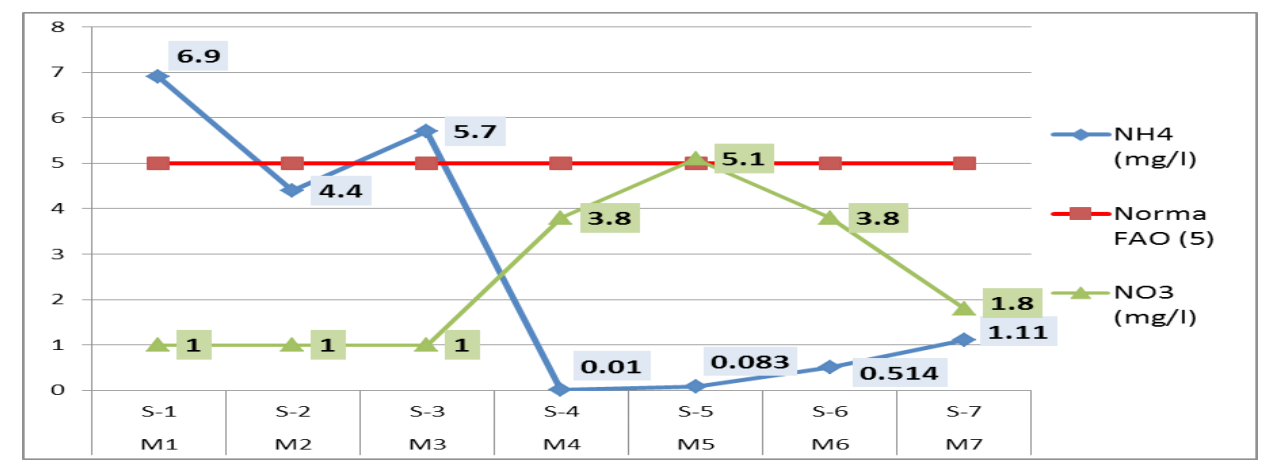

Figura 19. Comparación de $\mathrm{NH}_{4}$ y $\mathrm{NO}_{3}$ del humedal con Cartucho con las ECAs 
La figura 19 muestra el $\mathrm{NH}_{4}$ en la salida del humedal con Cartucho que disminuye a medida que el $\mathrm{NO}_{3}$ aumenta, debido al proceso de oxidación del $\mathrm{NH}_{4}$. Comparando con la norma, el $\mathrm{NO}_{3}$ que sale del humedal está por debajo de la norma, cuyo valor aceptable es $5 \mathrm{mg} / \mathrm{l}$.

\section{DISCUSIÓN}

En los trabajos de tratamiento de aguas residuales con humedales los porcentajes de remoción superan el 90\%; y comparados con nuestro trabajo, la remoción de la Demanda Bioquímica de Oxígeno (DBO) y la Demanda Química de Oxígeno(DQO) en la salida del humedal con Cartucho fue $68 \%$ y en el humedal con Cola de caballo fue $58 \%$, siendo menores comparándolos con resultados de trabajos similares de humedales que mostraron remociones de $72,84 \%$ en DBO y 76,85\% en DQO (Cantoral, 2015); esto por diversos factores que influyen en el funcionamiento de los humedales como la temperatura y la carga orgánica.

La remoción de SST tiene porcentajes de remoción de 71\% en el biodigestor, en el humedal con Cola de caballo 80\% y en el humedal con Cartucho 60\%, que en trabajos similares mostraron una remoción de SST de 79,68\% (Cantoral, 2015). En cuanto a los coliformes totales y fecales no se dio remoción alguna por el rol de degradación que desarrollan.

El Fosfato $\left(\mathrm{PO}_{4}\right)$, en el biodigestor se incrementa, en el humedal con Cola de caballo baja por la absorción rápida y en el humedal con Cartucho aumenta. En cuanto a los Nitritos $\left(\mathrm{NO}_{2}\right)$ y Nitratos $\left(\mathrm{NO}_{3}\right)$ en el Biodigestor baja; en el humedal con Cola de caballo aumenta por la poca absorción y en el humedal con Cartucho baja por la absorción en las plantas.

\section{CONCLUSIONES}

El uso de Unidades Básicas de Saneamiento (UBS) con humedales artificiales con especies nativas permite remover la materia orgánica biodegradable del agua residual en Tuyu - Ruri, mostrando remociones de $82 \%$ en $\mathrm{DBO}_{5}, 81 \%$ en DQO y $71 \%$ de SST.

La remoción de la $\mathrm{DBO}_{5}$ en la salida del humedal con Cola de caballo fue $58 \%$ y el de Cartucho 68\%, en ambos casos está por encima del valor aceptable de la norma. La remoción de la DQO en la salida del humedal con Cartucho fue 68\% y del humedal con Cola de caballo de 58\%. La remoción de SST en la salida del humedal con Cola de caballo fue $80 \%$ y del humedal con Cartucho $60 \%$, estando en ambos casos por debajo de la norma.

Los Coliformes totales y fecales no fueron removidos en todo el sistema, estando por encima del valor de la norma. El Fosfato $\left(\mathrm{PO}_{4}\right)$ en el humedal con Cartucho presenta una cantidad de 0,74 mg/l, en el humedal con Cola de caballo de 0,64 mg/l, estando por debajo de la norma. En cuanto al $\mathrm{NH}_{4}$ y $\mathrm{NO}_{3}$ en el humedal con Cola de caballo el $\mathrm{NH}_{4}$ se oxida rápidamente y en el humedal con Cartucho la oxidación es lenta, por ello 
la cantidad de $\mathrm{NO}_{3}$ en el humedal con Cola de caballo es alta, de 21,3 mg/l a diferencia del humedal con Cartucho que es baja, de 1,0 mg/1, en ambos casos están por debajo de los valores de la norma.

El uso de UBS con humedales artificiales si son eficientes; siendo el humedal con Cartucho el más eficiente, debido a la rápida adaptación de los microorganismos y las plantas.

\section{REFERENCIAS BIBLIOGRÁFICAS}

Cantoral, Rolando. 2015. «Tratamiento de aguas residuales grises domésticas con la especie «Paragüitas» Cyperus alternifolius en humedales artificiales, urbanización Zárate - San Juan de Lurigancho 2015. Facultad de Ingeniería - Universidad César Vallejo». <http://www.repositorio.ucv.edu.pe/bitstream/handle/UCV/118/ tito_cr.pdf?sequence $=1>$ [Consulta: 12-08-2017].

Delgadillo, Oscar et al. 2010. «Depuración de aguas residuales por medio de humedales artificiales». < https://core.ac.uk/download/pdf/48017573.pdf> [Consulta: 21-09-2017].

Perdana, M.; Sutanto, H. y Prihatmo, G. 2018. «Vertical Subsurface Flow (VSSF) constructed wetland for domestic wastewater treatment». <http://iopscience.iop. org/article/10.1088/1755-1315/148/1/012025/pdf> [Consulta: 25-10-2017]

Decreto Supremo. 2017. «Aprueban estándares de calidad ambiental (ECA) para agua y establecen disposiciones complementarias». <http://www.minam.gob.pe/ wp-content/uploads/2017/06/DS-004-2017-MINAM.pdf $>$ [Consulta: 06-072017].

DIGESA. 2008. «Estándares de calidad ambiental de agua grupo $\mathrm{N}^{\circ}$ 3: riego de vegetales y bebida de animales». <http://www.digesa.minsa.gob.pe/DEPA/informes_tecnicos/GRUPO\%20DE\%20USO\%203.pdf> [Consulta: 06-07-2017].

Vymazal, Jan. 2015. «Accumulation of heavy metals in aboveground biomass of Phragmites australis in horizontal flow constructed wetlands for wastewater treatment: A review». <https://www.sciencedirect.com/science/article/pii/ S1385894716300171> [Consulta: 06-07-2017].

Recepción: 26/09/2018

Aceptación: 30/11/2018

\section{Correspondencia}

Polo Salazar, Rosario Adriana

rosario_polo@hotmail.com 